\title{
NOTE ON MONOIDAL MONADS
}

\author{
B. J. DAY \\ (Received 10 May 1976)
}

\begin{abstract}
The representation theory of categories is used to embed each promonoidal monad in a monoidal biclosed monad. The existence of a promonoidal structure on the ordinary EilenbergMoore category generated by a promonoidal monad is examined. Several results by previous authors (notably A. Kock and F. E. J. Linton) are reproved and extended.
\end{abstract}

\section{Introduction}

The aim of this note is to employ the representation theory of categories to discuss the theories of monoidal monads and closed monads under the common concept of a promonoidal monad. We show that each promonoidal monad is embedded in a monoidal closed monad and we extend several results by previous authors (notably Kock (1971a) and Linton (1969)) on this topic.

In $\$ 1$ we discuss promonoidal categories. These, together with promonoidal functors and promonoidal natural transformations, form a 2-category called Pmon. Whilst colimits in Pmon are easily computed, the existence of limits is generally difficult to determine.

In $\$ 2$ we discuss a sufficient condition for the existence of a "trace" promonoidal structure on a full subcategory of a given promonoidal category.

In $\$ 3$ we use the result of $\$ 2$ to discuss promonoidal monads and whether the usual Eilenberg-Moore category of the underlying ordinary monad can be enriched to give the Eilenberg-Moore construction in Pmon.

In conclusion we briefly mention that we hope to develop, in a further article, some generalisations which apply to algebraic structures which generalise the concept of a monoidal category. These structures are the algebras for a doctrine generated by a suitable type of club of operations (as described in Kelly (1974)).

Unless otherwise stated the unexplained notations and terminology of 
this article are those of Day (1970a), Day and Kelly (1969), Dubuc (1970), Eilenberg and Kelly (1966), and Street (1972). We shall assume familiarity with the various aspects of the representation theorem and the theory of $\mathscr{V}$-monads for a suitable symmetric monoidal closed ground category $\mathscr{V}$.

\section{Promonoidal categories}

It is supposed throughout the article that, unless otherwise stated, all categorical algebra is relative to a suitably complete and cocomplete symmetric monoidal closed category $\mathscr{V}=(\mathscr{V}, \otimes, I, \cdots)$.

We recall from Day (1970a) that a promonoidal category (over $\mathscr{V}$ ) consists of data $(\mathscr{A}, P, J, \alpha, \rho, \lambda)$ where $\mathscr{A}$ is a (small) category,

$$
\begin{aligned}
& P: \mathscr{A}^{\mathrm{oP}} \otimes \mathscr{A}^{\mathrm{op}} \otimes \mathscr{A} \rightarrow \mathscr{V} \\
& J: \mathscr{A} \rightarrow \mathscr{V}
\end{aligned}
$$

are functors, and

$$
\begin{aligned}
& \alpha: P(A X-) \circ P\left(A^{\prime} A^{\prime \prime} X\right) \cong P\left(A A^{\prime} X\right) \circ P\left(X A^{\prime \prime}-\right) \\
& \rho: J X \circ P(A X-) \cong \mathscr{A}(A-) \\
& \lambda: J X \circ P(X A-) \cong \mathscr{A}(A-)
\end{aligned}
$$

are natural isomorphisms satisfying suitable coherence axioms (namely axioms PC1 and PC2 of Day (1970a) where "o" denotes profunctor composition. The promonoidal structure has a symmetry $\sigma$ if

$$
\sigma: P\left(A A^{\prime}-\right) \cong P\left(A^{\prime} A-\right)
$$

is a natural isomorphism satisfying axioms $\mathrm{PC} 3\left(\sigma^{2}=1\right)$ and PC4 of Day (1970a).

The convolution $[\mathscr{A}, \mathscr{V}]$ of a promonoidal category $\mathscr{A}$ with the ground category $\mathscr{V}$ is the monoidal biclosed structure on the functor category $[\mathscr{A}, \mathscr{V}]$ given by the formulas:

$$
\begin{aligned}
F \otimes G & =\int^{A A^{\prime}} F A \otimes G A^{\prime} \otimes P\left(A A^{\prime}-\right) \\
F \backslash G & =\int_{A A^{\prime}}\left[P\left(A-A^{\prime}\right) \otimes F A, G A^{\prime}\right] \\
G / F & =\int_{A A^{\prime}}\left[P\left(-A A^{\prime}\right) \otimes F A, G A^{\prime}\right] .
\end{aligned}
$$

A promonoidal functor $\Phi=(\phi, \widehat{\phi}, \phi): \mathscr{A} \rightarrow \bar{A}$ between promonoidal categories $\mathscr{A}$ and $\bar{A}$ comprises a functor $\phi: \mathscr{A} \rightarrow \bar{A}$ and natural transformations 


$$
\begin{aligned}
& \phi: P\left(A A^{\prime} A^{\prime \prime}\right) \rightarrow \bar{P}\left(\phi A, \phi A^{\prime}, \phi A^{\prime \prime}\right) \\
& \phi: J A \rightarrow \bar{J} \phi A
\end{aligned}
$$

satisfying axioms which give the functor

$$
[\phi, 1]:[\bar{A}, \mathscr{V}] \rightarrow[\mathscr{A}, \mathscr{V}]
$$

the structure of a monoidal closed functor on the convolution.

Given promonoidal functors $\Phi$ and $\Psi$ from $\mathscr{A}$ to $\overline{\mathscr{A}}$, a promonoidal natural transformation $\eta: \Phi \rightarrow \Psi: \mathscr{A} \rightarrow \overline{\mathscr{A}}$ is a natural transformation $\eta: \phi \rightarrow \psi$ which satisfies the following two axioms:

PN1. The following diagram commutes:

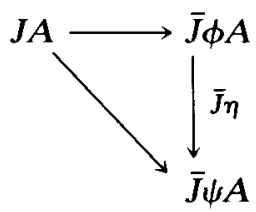

PN2. The following diagram commutes:

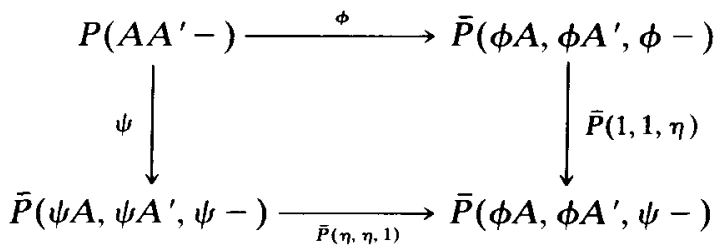

Thus we obtain the 0 -cells, 1-cells, and 2-cells of a 2-category which we call Pmon. It is easily seen to contain the 2-categories of monoidal categories and associative closed categories as full sub-2-categories.

The cocompleteness of Pmon is an easy consequence of the fact that the convolution process maps an ordinary 2-colimit of promonoidal categories to the corresponding limit of monoidal biclosed categories of the form $[\mathscr{A}, \mathscr{V}]$. Our aim is to find whether the Eilenberg-Moore construction in Cat for a promonoidal monad can be enriched to yield the Eilenberg-Moore construction in Pmon. Our results go a little further than those of previous authors.

We conclude this section with two remarks. Firstly, one can easily define symmetric promonoidal functors and thus obtain a sub-2-category of Pmon. However we shall not deal with this situation explicitly in the sequel. Secondly, we have assumed that all promonoidal categories are "small" relative to $\mathscr{V}$. The change-of- $\mathscr{V}$-universe process described in Day (1973) provides a convenient method of bypassing this apparent restriction. 


\section{Promonoidal traces}

Given a full embedding $M: \mathscr{A} \rightarrow \overline{\mathscr{A}}$ of a category $\mathscr{A}$ into a promonoidal category $\overline{\mathscr{A}}$ we can ask for conditions under which $\overline{\mathscr{A}}$ induces a trace promonoidal structure on $\mathscr{A}$. For our purpose it will suffice to consider the case in which $\bar{A}$ is a monoidal category which we call $\mathscr{B}$. With this data we have:

2.1 Proposirion. (strong trace conditions). The category $\mathscr{A}$ is promonoidal with respect to the functors

$$
\begin{aligned}
P\left(A, A^{\prime}, A^{\prime \prime}\right) & =\mathscr{B}\left(M A \otimes M A^{\prime}, M A^{\prime \prime}\right) \\
J A & =\mathscr{B}(I, M A)
\end{aligned}
$$

if the following canonical morphisms are isomorphisms for all $A, A^{\prime}, A^{\prime \prime} \in \mathscr{A}$ :

$$
\begin{aligned}
z_{1}: \mathscr{B}(I, M X) \circ \mathscr{B}(M X \otimes M A, M-) & \rightarrow \mathscr{B}(I \otimes M A, M-) \\
z_{1}^{*}: \mathscr{B}(I, M X) \circ \mathscr{B}(M A \otimes M X, M-) & \rightarrow \mathscr{B}(M A \otimes I, M-) \\
z_{2}: \mathscr{B}\left(M A \otimes M A^{\prime}, M X\right) \circ \mathscr{B}(M X \otimes & \left.M A^{\prime \prime}, M-\right) \\
& \rightarrow \mathscr{B}\left(\left(M A \otimes M A^{\prime}\right) \otimes M A^{\prime \prime}, M-\right) \\
z_{2}^{*}: \mathscr{B}\left(M A^{\prime} \otimes M A^{\prime \prime}, M X\right) \circ \mathscr{B}(M A \otimes & M X, M-) \\
& \rightarrow \mathscr{B}\left(M A \otimes\left(M A^{\prime} \otimes M A^{\prime \prime}\right), M-\right) .
\end{aligned}
$$

If these conditions are satisfied then $M$ becomes a promonoidal functor.

Proof. The isomorphisms $\alpha, \rho$, and $\lambda$ are defined by requiring the following diagrams to commute:

$$
P\left(A A^{\prime} X\right) \circ P\left(X A^{\prime \prime}-\right) \stackrel{\alpha}{\longrightarrow} P\left(A^{\prime} A^{\prime \prime} X\right) \circ P(A X-)
$$

$\mathscr{B}\left(M A \otimes M A^{\prime}, M X\right) \circ \mathscr{B}\left(M X \otimes M A^{\prime \prime}, M-\right) \quad \mathscr{B}\left(M A^{\prime} \otimes M A^{\prime \prime}, M X\right) \circ \mathscr{B}(M A \otimes M X, M-)$ $z_{2}\left\lfloor\mathscr{B}\left(M A \otimes M A^{\prime}, Y\right)^{\circ} \circ \mathscr{B}\left(Y \otimes M A^{\prime \prime}, M-\right) \stackrel{\grave{m}}{\rightarrow} \mathscr{B}\left(M A^{\prime} \otimes M A^{\prime \prime}, Y\right)^{\circ} \circ(M A \otimes Y, M-) \mid z_{2}^{*}\right.$

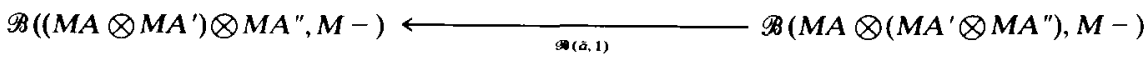

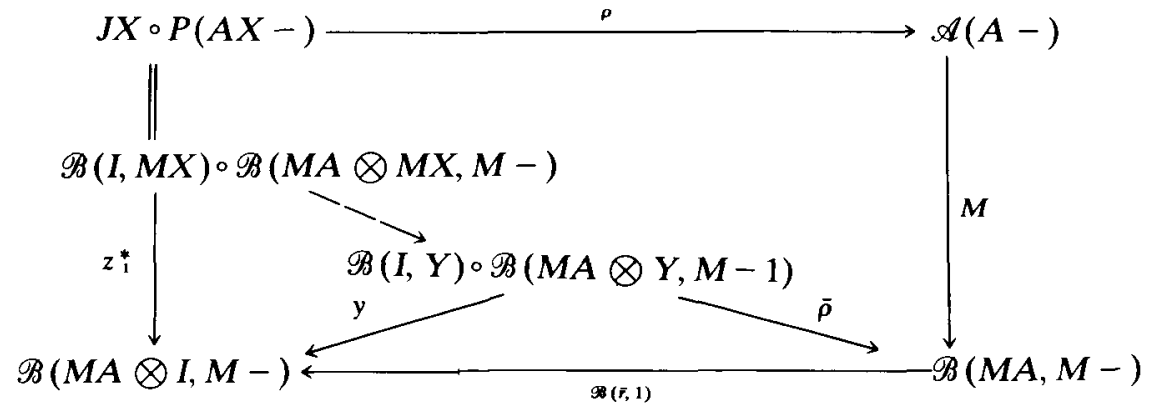




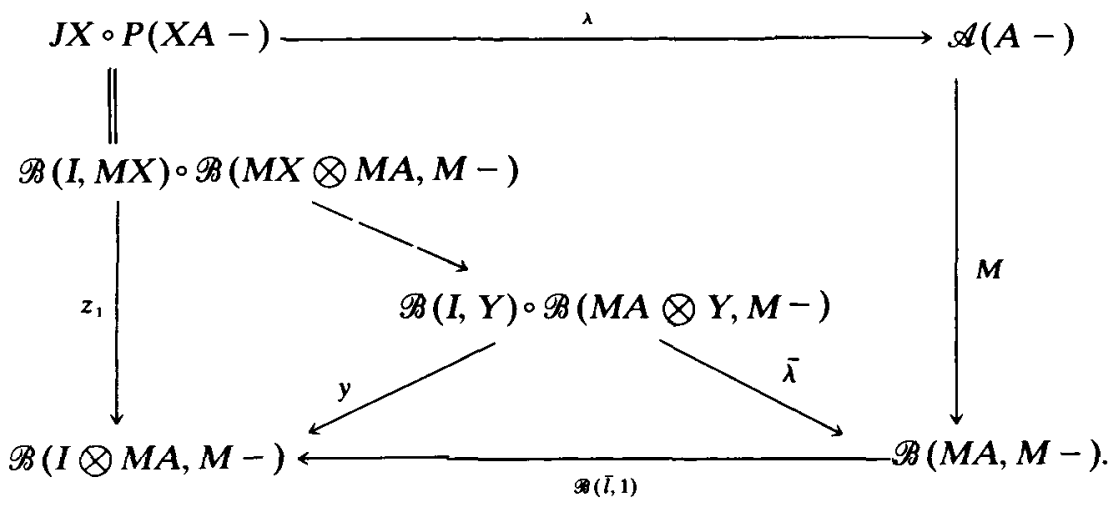

The verification of the axioms PC1 and PC2 (and, in the case of a symmetry, PC3 and PC4) is straightforward but tedious; the author has verified them in Day (1970b). The promonoidal functor axioms are easily verified for $\boldsymbol{M}$.

2.2 ExAmple. Suppose $\mathscr{A}$ is a small category and $\mathscr{F}=[\mathscr{A}, \mathscr{V}]$ has a monoidal biclosed structure. The full embedding $L=Y^{\text {op }}: \mathscr{A} \rightarrow \mathscr{F}^{\text {op }}$ satisfies the strong trace conditions. To see this we note that the following transformations are isomorphisms for all $F \in \mathscr{F}$ :

$$
\begin{aligned}
z: \mathscr{F}(L X, F) \circ \mathscr{F}\left(L A, L X \otimes L A^{\prime}\right) & \rightarrow \mathscr{F}\left(L A, F \otimes L A^{\prime}\right) \\
z^{*}: \mathscr{F}(L X, F) \circ \mathscr{F}\left(L A, L A^{\prime} \otimes L X\right) & \rightarrow \mathscr{F}\left(L A, L A^{\prime} \otimes F\right) .
\end{aligned}
$$

It is easily seen that this trace structure, when convoluted with $\mathscr{V}$, yields the original monoidal biclosed structure on $\mathscr{F}=[\mathscr{A}, \mathscr{V}]$ (to within a monoidal isomorphism of categories).

2.3 Example. Suppose $\mathscr{A}$ contains $I$ and is closed under the monoidal structure on $\mathscr{B}$. Then, by the representation theorem, the strong trace :onditions are satisfied.

2.4 Example. Suppose $\mathscr{B}$ is monoidal biclosed and $\mathscr{A}$ contains $I$ and is closed under the internal-hom functors of $\mathscr{B}$. Then, by the representation theorem, the strong trace conditions are satisfied.

2.5 Example. Suppose $\mathscr{V}$ is cartesian closed. A cocartesian monoidal structure on $\mathscr{B}$ (that is $\otimes=+$ ) leaves a trace on any full subcategory $\mathscr{A}$ of $\mathscr{B}$. One way to verify this is to observe that the resulting reflective embedding

$$
[M, 1] \dashv \underline{M}:[\mathscr{A}, \mathscr{V}] \rightarrow[\mathscr{B}, \mathscr{V}]
$$

is always cartesian monoidal. 
2.6 Example. More generally, suppose $M: \mathscr{A} \rightarrow \mathscr{B}$ is a full embedding and consider the reflective embedding:

$$
[M, 1] \dashv \underline{M}:[\mathscr{A}, \mathscr{V}] \rightarrow[\mathscr{B}, \mathscr{V}] .
$$

It can be shown (see Day (1974a) \$1) that this is a monoidal biclosed adjunction if and only if the following two canonical morphisms are isomorphisms for all $B, B^{\prime} \in \mathscr{B}$ and $A \in \mathscr{A}$ :

$$
\begin{aligned}
z: \mathscr{B}(B, M X) \circ \mathscr{B}\left(M X \otimes B^{\prime}, M A\right) & \rightarrow \mathscr{B}\left(B \otimes B^{\prime}, M A\right) \\
z^{*}: \mathscr{B}(B, M X) \circ \mathscr{B}\left(B^{\prime} \otimes M X, M A\right) & \rightarrow \mathscr{B}\left(B^{\prime} \otimes B, M A\right) .
\end{aligned}
$$

Thus the strong trace conditions are satisfied by suitable choice of $B, B^{\prime} \in \mathscr{B}$. Note that $\mathscr{B}$ could be replaced here by the monoidal full subcategory of $\mathscr{B}$ generated by the image of $\mathscr{A}$ under $M$.

2.7 Example. Suppose $\mathscr{B}$ is monoidal biclosed and $M \mid \dashv R: \mathscr{B} \rightarrow \mathscr{A}$ with unit $1 \rightarrow R M$ an isomorphism; thus $\mathscr{A}$ is coreflective in $\mathscr{B}$. Then, applying the representation theorem, the strong trace conditions are satisfied by $M$ if and only if the counits $M R(B \backslash M A) \rightarrow B \backslash M A$ and $M R(M A / B) \rightarrow M A / B$ are isomorphisms for suitable choice of $B \in \mathscr{B}$ and all $A \in \mathscr{A}$.

\section{Promonoidal monads}

We suppose that $\mathscr{A}$ is a fixed promonoidal category and that $\mathscr{T}=$ $(T, \mu, \eta)$ is a promonoidal monad on $\mathscr{A}$. Thus $\mathscr{T}$ is a monad in Pmon. This implies that $[\mathscr{T}, 1]$ is a monoidal biclosed monad on the convolution $[\mathscr{A}, \mathscr{V}]$.

The usual resolution of $\mathscr{T}$ into a Kleisli category $\mathscr{A}_{\mathscr{F}}$ and an EilenbergMoore category $\mathscr{A}^{\mathcal{F}}$ is denoted by the following diagram:

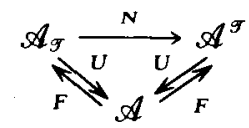

The functor $F: \mathscr{A} \rightarrow \mathscr{A}_{\mathscr{T}}$ gives the adjunction on $[\mathscr{A}, \mathscr{V}]$.

$$
[\mathscr{A}, \mathscr{V}] \underset{[F, 1]}{\stackrel{[U, 1]}{\rightleftarrows}}\left[\mathscr{A}_{\mathscr{T}}, \mathscr{V}\right] \text {. }
$$

Thus $\left[\mathscr{A}_{\mathscr{F}}, \mathscr{V}\right]$ is a monoidal biclosed category and $\bar{F}=[U, 1]$ preserves the identity and (finite) tensor products. From this we deduce that $\mathscr{A}_{\mathscr{T}}$ has a canonical promonoidal structure (see 2.2 Example) which we denote by $\left(P^{\prime}, J^{\prime}, \cdots\right)$. In fact, $\mathscr{A}_{\mathscr{S}}$ is now the Kleisli category of $\mathscr{T}$ in Pmon (cf. Street (1972)). We shall omit this verification and simply note the following: 
3.1 Proposition. $\quad P\left(A, A^{\prime}, U-\right) \cong P^{\prime}\left(F A, F A^{\prime},-\right)$ naturally in $A, A^{\prime} \in \mathscr{A}$.

PRoOF. This follows from the fact that $\bar{F}=[U, 1]$ preserves tensor products.

It is straightforward to check that $P^{\prime}$ is defined "on morphisms" as follows:

3.2 Proposition. If $f \in V \mathscr{A}_{\mathscr{G}}\left(F A^{\prime}, F A\right), g \in V \mathscr{A}_{\mathscr{g}}\left(F B^{\prime}, F B\right)$, and $h \in$ $V \mathscr{A}_{\mathcal{F}}\left(F C, F C^{\prime}\right)$ then the following diagram commutes:

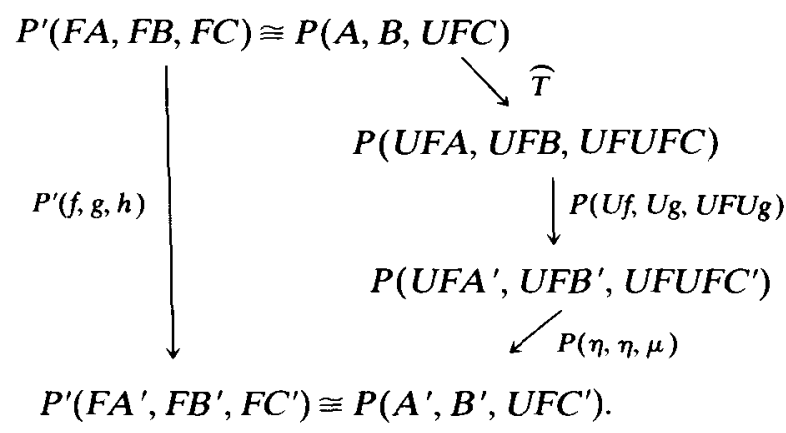

3.3 Remark. The symbol $U$ is omitted if no confusion seems likely.

Define $\mathscr{B}^{\text {op }} \subset[\mathscr{A}, \mathscr{V}]$ to be the full subcategory of $[\mathscr{A}, \mathscr{V}]$ comprising $J$ together with all tensor products of representable functors with each other (and with $J$ ). Define $\mathscr{B}_{\mathscr{F}}^{\text {op }} \subset\left[\mathscr{A}_{\mathscr{F}}, \mathscr{V}\right]$ similarly. Then, because $\bar{F}=[U, 1]$ preserves tensor products, we obtain an induced strong monoidal functor

$$
\bar{F}: \mathscr{B}^{\text {op }} \rightarrow \mathscr{B}_{\mathscr{T}}^{\text {op }}
$$

which is surjective (to within isomorphism) on objects. Thus there exists a monadic adjunction

$$
\overline{\bar{F}} \dashv[\bar{F}, 1]:\left[\mathscr{B}_{\mathscr{T}}^{\text {op }}, \mathscr{V}\right] \rightarrow\left[\mathscr{B}^{\text {op }}, \mathscr{V}\right]
$$

on the convolution $\left[\mathscr{B}^{\text {op }}, \mathscr{V}\right]$. Indeed, $\mathscr{B} \stackrel{\text { op }}{F}$ may be regarded as the "monoidal theory" of $\mathscr{T}$.

This gives the following summary diagram:

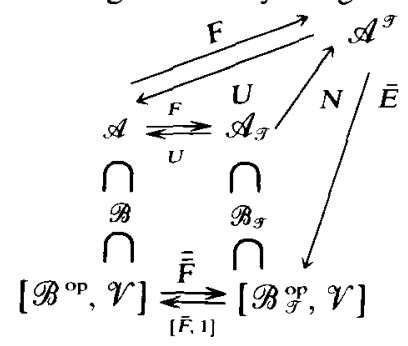


where $E: \mathscr{A}_{\mathscr{S}} \subset\left[\mathscr{B}_{\mathscr{J}}^{\circ}, \mathscr{V}\right]$ is "evaluation". The functor $\bar{E}$ is defined to be $\operatorname{Lan}_{N} E$. Thus, if $(A, \xi) \in \mathscr{A}^{\mathscr{T}}$ then $\bar{E}(A)$ is the coequaliser in $[\mathscr{B} \stackrel{\text { op }}{\mathcal{V}}, \mathscr{V}$ ] of the following pair of morphisms:

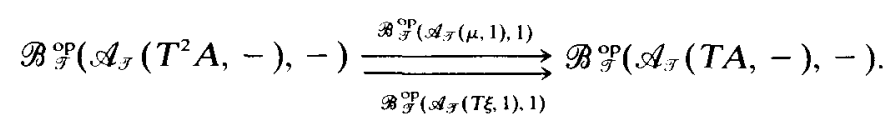

Alternatively,

$$
\bar{E}(A)=\int^{X} \mathscr{A}^{\mathscr{T}}(N K, A) \cdot E X
$$

The functor $\bar{E}$ is a fully faithfull extension of $E$ because $N$ is dense (= adequate) (see $\$ 5$, Appendix I). This gives the following representation of $\mathscr{T}$ :

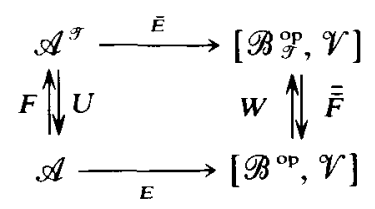

where $W=[\bar{F}, 1]$.

3.4 Proposition. $W \bar{E} \cong E U$ and $\bar{E} F \cong \overline{\bar{F}} E$.

Proof. $\bar{E}$ is defined at $(A, \xi)$ to be the coequaliser of the canonical pair:

$$
\mathscr{B}_{\mathscr{T}}^{\mathrm{op}}\left(\mathscr{A}_{\mathscr{T}}\left(T^{2} A,-\right),-\right) \rightrightarrows \mathscr{B}_{\mathscr{J}}^{\mathrm{op}}\left(\mathscr{A}_{\mathscr{F}}(T A,-),-\right)
$$

Thus, because $W$ preserves coequalisers, $W \bar{E}(A)$ is the coequaliser of the canonical pair:

$$
\left[\mathscr{A}_{\mathscr{T}}, \mathscr{V}\right]\left(\mathscr{A}_{\mathscr{T}}\left(T^{2} A,-\right), \vec{F}-\right) \rightrightarrows\left[\mathscr{A}_{\mathcal{T}}, \mathscr{V}\right]\left(\mathscr{A}_{\mathscr{T}}(T A,-), \vec{F}-\right)
$$

But $\bar{F}(H)=H U$ for all $H \in \mathscr{B}^{\mathrm{op}} \subset[\mathscr{A}, \mathscr{V}]$. Thus $W \bar{E}(A)(H)$ is the coequaliser of the canonical pair:

$$
H U\left(T^{2} A\right) \rightrightarrows H U(T A)
$$

Thus

$$
\begin{aligned}
W \bar{E}(A)(H) & \cong H U A \\
& \cong E(U A)(H) \text { for all } H \in \mathscr{B}^{\circ} .
\end{aligned}
$$

Secondly, 


$$
\begin{aligned}
\overline{\bar{F}} E(A) & \cong \overline{\bar{F}}\left(\mathscr{B}^{\mathrm{op}}(\mathscr{A}(A,-),-)\right) \\
& =\int^{\mathrm{x}} \mathscr{B}_{\mathscr{F}}^{\mathrm{op}}(\bar{F} X,-) \otimes \mathscr{B}^{\mathrm{op}}(\mathscr{A}(A-), X) \\
& \cong \mathscr{B}_{\mathscr{P}}^{\mathrm{op}}(\bar{F}(\mathscr{A}(A-)),-) \\
& =\mathscr{B}_{\mathscr{O}}^{\mathrm{op}}(\mathscr{A}(A, U-),-) \\
& \cong \mathscr{B}_{\mathscr{O}}^{\mathrm{op}}\left(\mathscr{A}_{\mathscr{T}}(F A,-),-\right) \\
& =E(F A) \cong \bar{E} F(A) \text { for all } A \in \mathscr{A} .
\end{aligned}
$$

We now ask for conditions under which the convolution structure $\mathscr{C}=\left[\mathscr{B}_{\mathscr{T}}^{\text {op }}, \mathscr{V}\right]$ induces a promonoidal trace on $\mathscr{A}^{\mathscr{F}}$.

3.5 ExAmple. Suppose the unit object $I$ of $\mathscr{C}$ is of the form $\vec{E} I$ for some object $I$ of $\mathscr{A}^{g}$ and suppose that there exists a functor $*$ from $\left(\mathscr{A}^{g}\right)^{\text {op }} \otimes \mathscr{A}^{g}$ to $\mathscr{A}^{\mathfrak{g}}$ such that

$$
\bar{E} A \otimes \bar{E} A^{\prime} \cong \bar{E}\left(A * A^{\prime}\right)
$$

for all $A, A^{\prime} \in \mathscr{A}^{\mathcal{J}}$. Then, by the representation theorem, the strong trace conditions are satisfied.

3.6 Example. Suppose $I \in \mathscr{C}$ is $\bar{E} I$ for some $I \in \mathscr{A}^{\mathscr{T}}$ and, for all $A, A^{\prime}, A^{\prime \prime} \in \mathscr{A}^{g}$ we have:

$$
\begin{aligned}
& \bar{E} A \backslash \bar{E} A^{\prime} \cong \bar{E}\left(A \backslash A^{\prime}\right) \\
& \bar{E} A^{\prime} / \bar{E} A \cong \bar{E}\left(A^{\prime} / A\right)
\end{aligned}
$$

for some $A \backslash A^{\prime}$ and $A^{\prime} / A$ in $\mathscr{A}^{g}$. Then, by the representation theorem, the strong trace conditions are satisfied.

3.7 Example. Suppose $C \otimes \bar{E} A=C+\bar{E} A$ whenever $C$ is of the form $I, \bar{E} A, \bar{E} A \otimes \bar{E} A^{\prime}$. Then, by the representation theorem, the strong trace conditions are satisfied.

It remains to determine formulas for the trace presentation. In examples 3.5 and 3.6 these turn out to be similar to formulas already discussed by earlier authors. We shall use the presentation of $\bar{E}(\boldsymbol{A})$ as the coequaliser of the canonical pair:

$$
E\left(T^{2} A\right) \underset{E(T \xi)}{\stackrel{E \mu}{\longrightarrow}} E(T A) .
$$

Suppose $A=(A, \xi), B=(B, \xi), C=(C, \xi)$ are $\mathscr{T}$-algebras.

3.8 Proposition. $C(E(F U A) \otimes E(F U B), \bar{E} C) \cong P(U A, U B, U C)$.

Proof. Firstly we compute $E(F U A) \otimes E(F U B)$ in $\mathscr{C}$. 
$E(F U A) \otimes E(F U B)$

$$
=\int^{X, Y} \mathscr{B}_{F}^{\mathrm{op}}\left(\mathscr{A}_{\mathscr{F}}(F A,-), X\right) \otimes \mathscr{B}_{F}^{\mathrm{op}}\left(\mathscr{A}_{\mathcal{F}}(F B,-), Y\right) \otimes \mathscr{B}_{F}^{\mathrm{og}}(X \otimes Y,-)
$$

by definition of the convolution,

$$
\cong \mathscr{B} \stackrel{\circ}{F}\left(\mathscr{A}_{\mathscr{F}}(F A,-) \otimes \mathscr{A}_{\mathscr{T}}(F B,-),-\right)
$$

by the representation theorem. Thus

$$
\begin{aligned}
\mathscr{C}(E(F U A) \otimes E(F U B), \bar{E} C) & \cong \mathscr{C}\left(\mathscr{B} \text { of }\left(\mathscr{A}_{F}(F A,-) \otimes \mathscr{A}_{\mathscr{F}}(F B,-),-\right), \bar{E} C\right), \\
& \cong \bar{E}(C)\left(\mathscr{A}_{\mathscr{F}}(F A,-) \otimes \mathscr{A}_{\mathscr{F}}(F B,-)\right)
\end{aligned}
$$

by the representation theorem. By definition of $\bar{E}(C)$ this object is the coequaliser of the two canonical morphisms from

$$
\mathscr{B}_{F}^{\text {op }}\left(\mathscr{A}_{\mathscr{F}}(\text { FUFUC, }-), \quad \mathscr{A}_{\mathcal{F}}(F U A,-) \otimes \mathscr{A}_{\mathscr{S}}(F U B,-)\right)
$$

to

$$
\mathscr{B}_{F}^{\circ}\left(\mathscr{A}_{\mathcal{F}}(F U C,-), \mathscr{A}_{\mathcal{F}}(F U A,-) \otimes \mathscr{A}_{\mathcal{F}}(F U B,-)\right) \text {. }
$$

Thus, by the representation theorem, the object

$$
\bar{E}(C)\left(\mathscr{A}_{\mathscr{T}}(F U A,-) \otimes \mathscr{A}_{T}(F U B,-)\right)
$$

is the coequaliser of the two canonical morphisms from

$$
\begin{aligned}
\left(\mathscr{A}_{\mathcal{F}}(F U A,-)\right. & \left.\otimes \mathscr{A}_{\mathcal{F}}(F U B,-)\right)(F U F U C) \\
& \cong P^{\prime}(F U A, F U B, F U F U C) \text { by definition of } \mathrm{P}^{\prime}, \\
& \cong P(U A, U B, U F U F U C) \text { by } 3.1 \text { Proposition, }
\end{aligned}
$$

to

$$
\begin{aligned}
\left(\mathscr{A}_{\mathscr{T}}(F U A,-)\right. & \left.\otimes \mathscr{A}_{\mathcal{F}}(F U B,-)\right)(F U C) \\
& \cong P^{\prime}(F U A, F U B, F U C) \text { by definition of } P^{\prime}, \\
& \cong P(U A, U B, U F U C) \text { by } 3.1 \text { Proposition. }
\end{aligned}
$$

Thus, because the canonical pair

\section{UFUFUC $\Longrightarrow U F U C$}

is a split (absolute) coequaliser, we have

$$
\bar{E}(C)\left(\mathscr{A}_{\mathcal{T}}(F U A,-) \otimes \mathscr{A}_{\mathcal{T}}(F U B,-)\right) \cong P(U A, U B, U C)
$$

on objects.

Note also that $\mathscr{C}(I, \bar{E}(C)) \cong J U C$.

A direct description of the trace on $\mathscr{A}^{\mathfrak{s}}$ can now be given in terms of $P$ alone. By 3.8 Proposition we know that $\mathscr{C}(\bar{E} A \otimes \bar{E} B, \bar{E} C)$ is the joint equaliser of the two canonical pairs: 


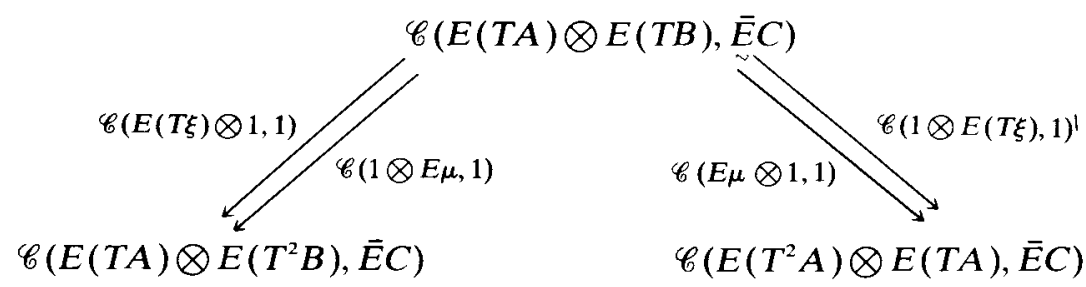

We denote this equaliser by $\bar{P}(A, B, C)$; it is easily seen to be functorial in each variable such that the inclusion $\bar{P} \rightarrow P$ is natural in these variables.

3.9 Proposition. $\bar{P}(A, B, C)$ is the joint equaliser of the following two pairs of morphisms:

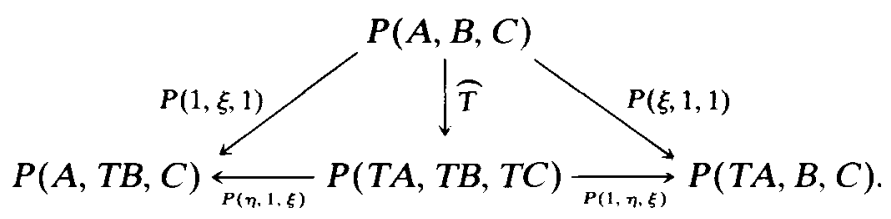

Proof. Consider the following diagram:

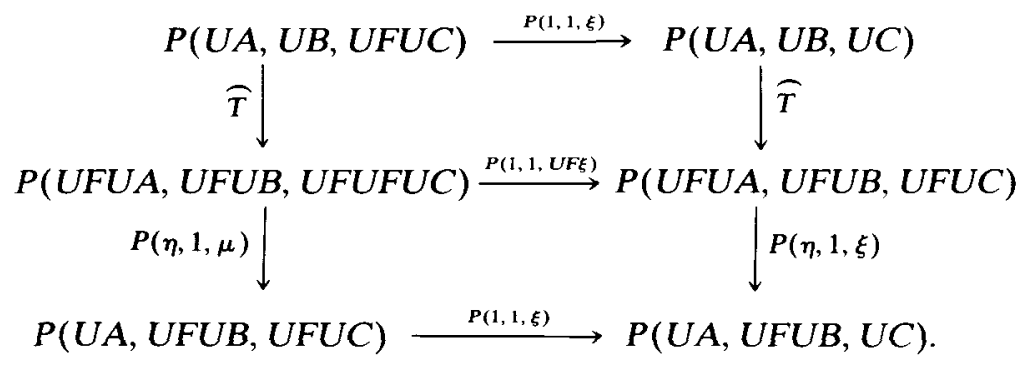

The upper half commutes by naturality of $\overparen{T}$, while the lower half commutes by an axiom on $\xi$. By 3.2 Proposition, this implies that the following diagram commutes:

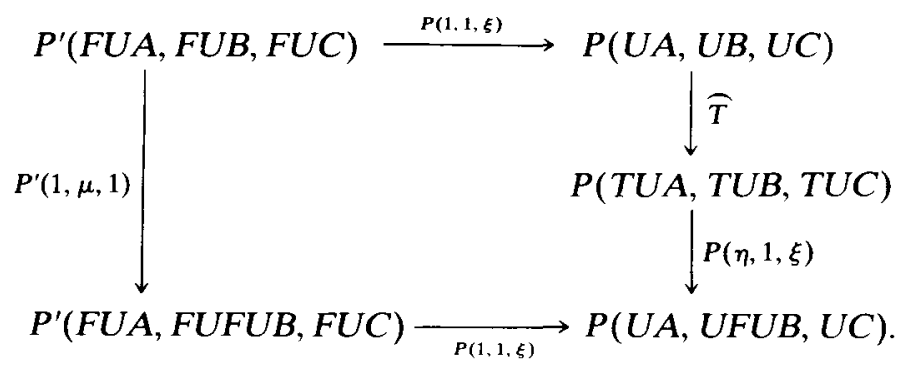

Thus $\mathscr{C}\left(1 \otimes E_{\mu}, 1\right)=P(\eta, 1, \xi) \cdot \overparen{T}$. Similarly $\mathscr{C}(E \mu \otimes 1,1)=P(1, \eta, \xi) \cdot \overparen{T} . \square$ 
3.10 Proposition. $\bar{P}(A, B, C)$ is the equaliser of the following pair of morphisms:

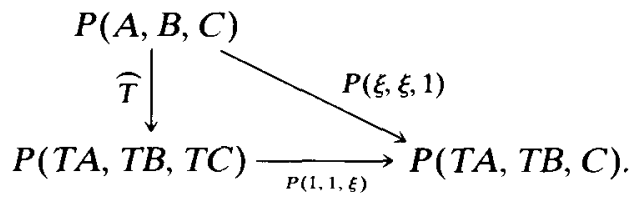

Proof. On composing this triangle with $P(\eta, 1,1)$ and $P(1, \eta, 1)$ we obtain the two pairs of morphisms respectively. Conversely, suppose a morphisms equalises both of the given pairs of morphisms. Consider the following diagram:

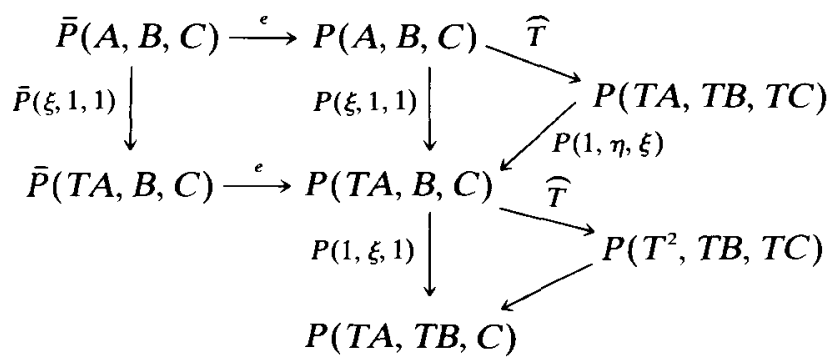

By naturality of $e$ we have that

$$
P(\xi, \xi, 1) e=P(\eta, 1, \xi) \cdot \widehat{T} \cdot P(1, \eta, \xi) \cdot \widehat{T} \cdot e .
$$

By naturality of $\widehat{T}$ we have

$$
\widehat{T} \cdot P(1, \eta, \xi)=P(1, T \eta, T \xi) \cdot \widehat{T} .
$$

Thus

$$
\begin{aligned}
& P(\eta, 1, \xi) \cdot \widehat{T} \cdot P(1, \eta, \xi) \cdot \widehat{T} \\
&=P(\eta, 1, \xi) \cdot P(1, T \eta, T \xi) \cdot \widehat{T} \cdot \widehat{T} \\
&=P(\eta, 1, \xi) \cdot P(1, T \eta, \mu) \cdot \widehat{T} \cdot \widehat{T} \\
&=P(\eta, 1, \xi) \cdot P(1, T \eta, 1) \cdot P(1,1, \mu) \cdot \widehat{T} \cdot \widehat{T} \\
&=P(\eta, 1, \xi) \cdot P(1, T \eta, 1) \cdot P(\mu, \mu, 1) \cdot \widehat{T}
\end{aligned}
$$

by promonoidal naturality of $\mu$,

$$
=P(1,1, \xi) \cdot \widehat{T} \text {. }
$$

Thus $e$ equalises $P(\xi, \xi, 1)$ and $(P 1,1, \xi) \overparen{T,}$ as required.

The trace structure on $\mathscr{A}^{\mathscr{T}}$ measures the extent to which 
$\xi: T \rightarrow 1: \mathscr{A} \rightarrow \mathscr{A}$ fails to be a promonoidal natural transformation. We conclude this section by noting that if the trace of $\mathscr{C}$ exists on $\mathscr{A}^{g}$ then $\mathscr{A}^{g}$ is the Eilenberg-Moore construction in Pmon (see Street (1972)).

We know that $U$ and $U \varepsilon$ are promonoidal. Suppose

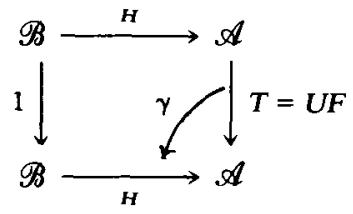

is a left lax transformation in Pmon. Then, using the fact that $H$ lifts to a left lax transformation $\delta$ in cat, we obtain $R$ as a functor:

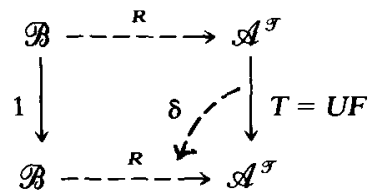

where $R B=H B$ and $\delta=\gamma$. It remains to verify that $(R, \delta)$ is promonoidal. Because $U$ is $\mathscr{V}$-faithful it suffices to construct $\widehat{R}$ and $R \cdot$ If $(\mathscr{B}, Q, K, \cdots)$ denotes the promonoidal structure on $\mathscr{B}$ then the following diagram commutes by promonoidal naturality of $\gamma: T H \rightarrow H$.

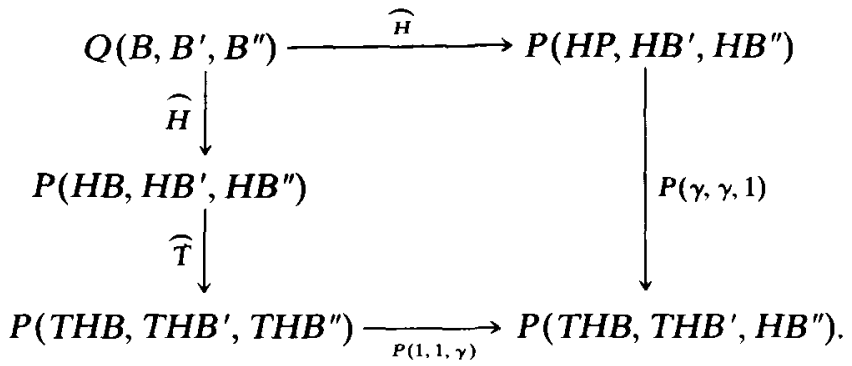

Thus $\widehat{H}$ factors through $\bar{P}\left(H B, H B^{\prime}, H B^{\prime \prime}\right)$. Similarly $H$ factors through $\bar{J}(H B)$.

\section{Examples; monoidal and biclosed monads}

There are two cases of special interest.

4.1 Example. Suppose that the initial promonoidal category $\mathscr{A}$ is monoidal; thus $P(A, B, C)=\mathscr{A}(A \otimes B, C)$. In this case $\bar{P}(A, B, C)$ is the equaliser of the pair: 


$$
\mathscr{A}(A \otimes B, C \overbrace{\widehat{T}}^{\stackrel{\mathscr{A}(\xi \otimes \xi, 1)}{\longrightarrow}} \mathscr{A}(T A \otimes T B, C)
$$

This corresponds to the pair:

$$
\mathscr{A}^{\mathcal{T}}(F(A \otimes B), C) \stackrel{\mathscr{A}_{\mathrm{T}}{ }^{g}(F(\xi \otimes \xi), 1)}{\longrightarrow} \mathscr{A}^{\mathcal{G}}(F(T A \otimes T B), C)
$$

Thus, by the representation theorem, $\mathscr{A}^{\mathscr{T}}$ is monoidal if the coequaliser in $\mathscr{A}^{\mathscr{T}}$ of each reflective pair of the form:

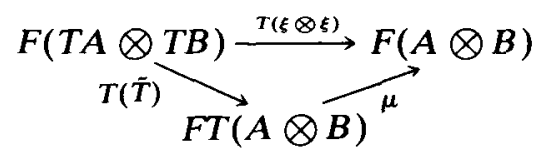

exists in $\mathscr{A}^{\mathcal{T}}$ and is preserved by the embedding $E U$ of $\mathscr{A}^{\mathscr{T}}$ in $\left[\mathscr{B}{ }^{\mathrm{op}}, \mathscr{V}\right]_{z^{\circ}}$ described in Appendix II and Day (1974b) $\$ 2$.

4.2 Example. Consider the case of $\mathscr{A}$ biclosed:

$$
P(A, B, C) \cong \mathscr{A}(B, A \mid C) \cong \mathscr{A}(A, C / B) .
$$

A canonical map $\tau: T(A \backslash B) \rightarrow T A \backslash T B$ is defined by means of the composite:

$$
\begin{aligned}
I \stackrel{\widehat{i}}{\longrightarrow} \mathscr{A}(A|B, A| B) & \cong P(A, A \mid B, B) \stackrel{\widehat{T}}{\longrightarrow} P(T A, T(A \backslash B), T B) \\
& \cong \mathscr{A}(T(A \backslash B), T A \backslash T B)
\end{aligned}
$$

and, dually, $\tau^{*}: T(B / A) \rightarrow T B / T A$ is defined.

4.3 Proposition. If $A$ and $B$ are $\mathscr{T}$-algebras then $A \backslash B$ and $B / A$ are $\mathscr{T}$-algebras with respect to the "evaluationwise" structures defined by

$$
\xi_{A \backslash B}: T(A \backslash B) \stackrel{r}{\longrightarrow} T A \backslash T B \stackrel{\eta ! \xi}{\longrightarrow} A \backslash B
$$

and

$$
\xi_{B / A}: t(B / A) \stackrel{r^{*}}{\longrightarrow} T B / T A \stackrel{\xi / \pi}{\longrightarrow} B / A
$$

respectively. 
Proof. Only $\xi_{A \backslash B}$ is explained since $\xi_{B / A}$ follows by duality. For the first algebra axiom on $\xi_{A \mid B}$ we have:

$$
\mathscr{A}(1, \eta \backslash \xi) \cdot \mathscr{A}(\eta, 1) \cdot \widehat{T} \cdot j: I \rightarrow \mathscr{A}(A \backslash B, A \mid B)
$$

equals

$$
\begin{aligned}
\mathscr{A}(1, & 1 \backslash \xi) \cdot \mathscr{A}(\eta, \eta \backslash 1) \cdot \widehat{T} \cdot j \\
& =\mathscr{A}(1,1 \backslash \xi) \cdot \mathscr{A}(1,1 \backslash \eta) \cdot j \text { by promonoidal naturality of } \eta \\
& =j \text { because } B \text { is an algebra. }
\end{aligned}
$$

The second algebra axiom for $A \backslash B$ is verified by showing

$$
(\eta \backslash \xi) \cdot \tau \cdot T(\eta \backslash \xi) \cdot T \tau=(\eta \backslash \xi) \cdot \tau \cdot \mu
$$

But

$$
\tau \cdot T(\eta \backslash \xi)=(T \eta \backslash T \xi) \cdot \tau \quad \text { by naturality of } \tau \text {, }
$$

and

$$
(\eta \backslash \xi) \cdot(T \eta \backslash T \xi)=(\eta \backslash \xi) \cdot\left(T_{\eta} \backslash \mu\right) \text { because } B \text { is an algebra }
$$

Thus we require

$$
\tau \cdot \mu=(T \eta \backslash \mu) \cdot \tau \cdot T \tau
$$

This follows from promonoidal naturality of $\mu$ because:

$$
\begin{aligned}
\mathscr{A}(1, T \eta \backslash \mu) \cdot \widehat{T} \cdot \widehat{T} & =\mathscr{A}(1, T \eta \backslash 1) \cdot \mathscr{A}(1,1 \backslash \mu) \cdot \widehat{T} \cdot \widehat{T} \\
& =\mathscr{A}(\mu, \mu \backslash 1) \cdot \widehat{T} .
\end{aligned}
$$

A subalgebra $A \Downarrow B$ of $A \backslash B$ is defined to be the equaliser of the iollowing pair of morphisms:

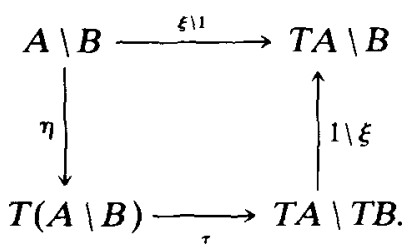

4.4 Proposition. $\bar{P}(A, B, C) \cong \mathscr{A}^{g}(B, A \Downarrow C)$ for all $A, B, C \in \mathscr{A}^{g}$.

Proof. Consider the following diagram: 


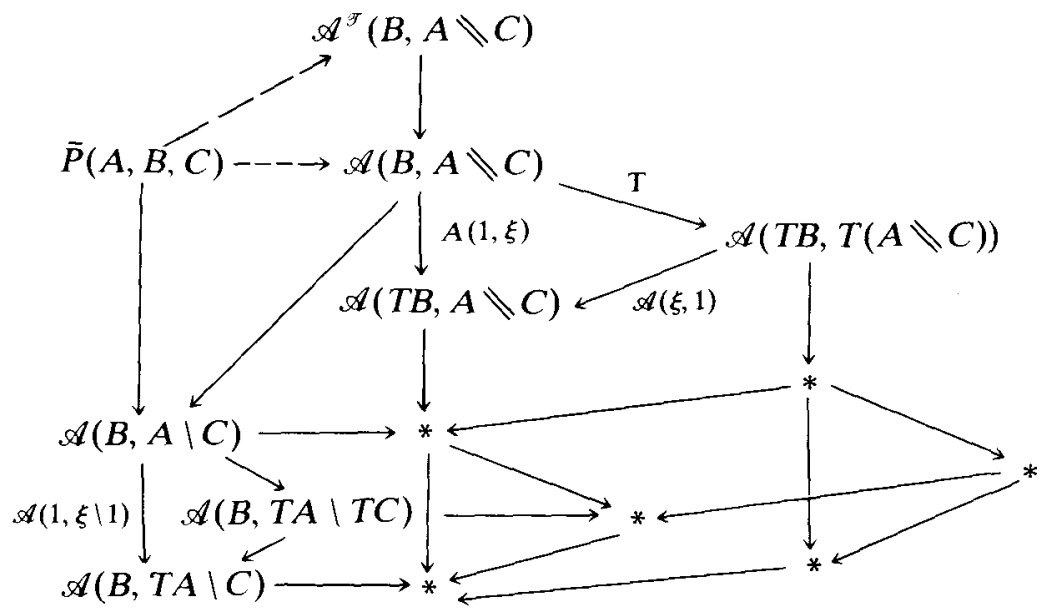

To show that the monomorphism from $\bar{P}(A, B, C)$ to $\mathscr{A}(B, A \backslash C)$ factors through $\mathscr{A}(B, A \rrbracket C)$ we have:

$$
\mathscr{A}(1,1 \backslash \xi) \cdot \mathscr{A}(1, \tau \eta)=\mathscr{A}(1,1 \backslash \xi) \cdot \mathscr{A}(\eta, 1) \cdot \widehat{T}
$$

by definition of $\tau$,

$$
=\mathscr{A}(\eta, 1) \cdot \mathscr{A}(1,1 \backslash \xi) \cdot \widehat{T}
$$

which is equalised with $\mathscr{A}(\eta, 1) \cdot \mathscr{A}(\xi, \xi \backslash 1)$ by $\bar{P}(A, B, C)$, as required. To show that the resultant monomorphism now factors through $\mathscr{A}^{\mathscr{T}}(B, A \rrbracket C)$ we consider the above diagram. A simple diagram chase reduces the requirement to commutativity of the following diagram:

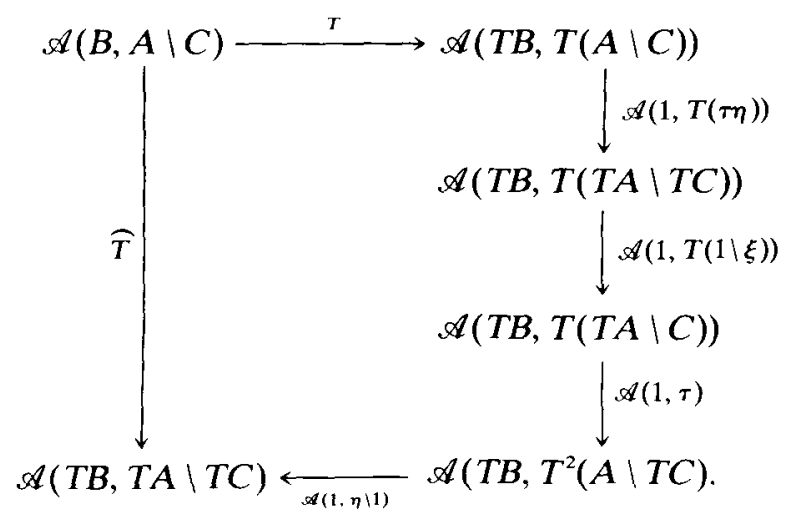

This diagram commutes because the representation theorem applied to $B \in \mathscr{A}$ gives the following diagram: 


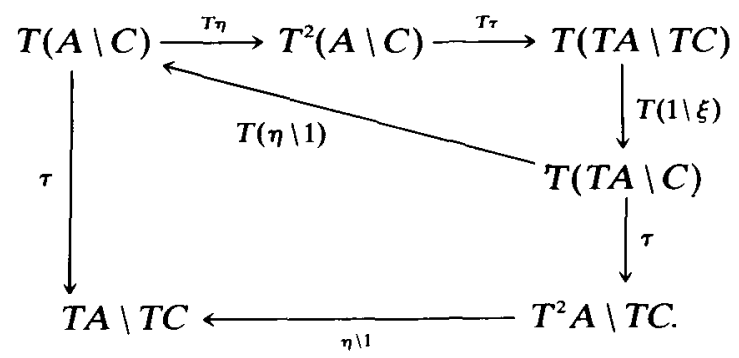

where the lower part commutes by naturality of $\tau$ while the upper part commutes because $\xi_{A \backslash C}=(\eta \backslash \xi) \tau$ is an algebra structure on $A \backslash C$. Conversely, we can follow the same lines and show that $\mathscr{A}^{g}(B, A \Downarrow C)$ equalises the following pair:

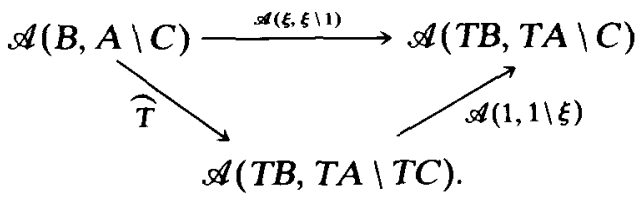

Thus $\vec{P}(A, B, C) \cong \mathscr{A}^{\mathscr{T}}(B, A \Downarrow C)$ and, similarly, $\bar{P}(A, B, C) \cong \mathscr{A}(A, C \Downarrow B)$.

The net result is that $\mathscr{A}^{\mathscr{T}}$ is biclosed if $\mathscr{A}$ is biclosed and the necessary equalisers exist in $\mathscr{A}$ and are preserved by the embedding $E$ of $\mathscr{A}$ in $\left[\mathscr{B}^{\text {op }}, \mathscr{V}\right]_{z^{0}}$ described in Appendix II and Day (1974b) $\$ 2$.

4.5 REMARK. In order that a biclosed promonoidal structure $(P, J, \alpha, \rho, \lambda)$ on $\mathscr{A}$ be associative in the sense that $\alpha$ be an isomorphism it is necessary and sufficient that the functors $-1-$ and $-/-$ be associative in the sense that the natural transformation with components $(A \backslash B) / C \rightarrow A \backslash(B / C)$ be an isomorphism. It is clear that a symmetric closed structure is associative in this sense. Moreover, it is straightforward to verify that the functors $-\mathbb{-}$ - and $-\mathscr{Z}$ - constructed in this section are associative if $-1-$ and $-/-$ are associative.

\section{Appendix I}

We recall several features of Kan extensions. Suppose $\mathscr{C}=[\mathscr{F}, \mathscr{V}]$ is a functor category where $\mathscr{F} \subset[\mathscr{A}, \mathscr{V}]$ and $\mathscr{F}$ is small relative to $\mathscr{V}$. Moreover, suppose $E: \mathscr{A} \rightarrow \mathscr{C}$ is "evaluation", $N: \mathscr{A} \rightarrow \mathscr{B}$ is a functor, and $\bar{E}$ is the left Kan extension of $E$ along $N$. 


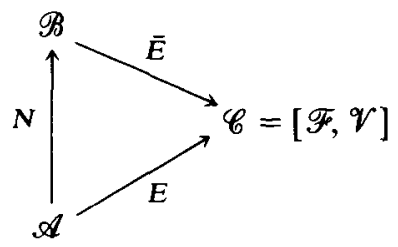

$$
\begin{aligned}
\bar{E}(B)=\int^{A} \mathscr{B}(N A, B) \cdot E A \\
\bar{E}\left(N A^{\prime}\right)=\int^{A} \mathscr{B}\left(N A, N A^{\prime}\right) \cdot E A \\
\cong \int^{A} \mathscr{A}\left(A, A^{\prime}\right) \cdot E A \text { if } N \text { is fully faithful, } \\
\cong E A^{\prime} \text { by the representation theorem. }
\end{aligned}
$$

We note that $\bar{E}$ is fully faithful if and only if $N$ is dense. If $F \in \mathscr{F}$ and $A \in \mathscr{A}$ then

$$
\begin{aligned}
E A(F) & =F A \\
& \cong \mathscr{F}(\mathscr{A}(A,-), F) \text { by the representation theorem, }
\end{aligned}
$$

thus

$$
\begin{aligned}
{[\mathscr{F}, \mathscr{V}](E A, F] } & =[\mathscr{F}, \mathscr{V}](\mathscr{F}(\mathscr{A}(A,-),-), F) \\
& \cong \mathrm{F}(\mathscr{A}(\mathrm{A},-))
\end{aligned}
$$

by the representation theorem. From this we may deduce:

$$
\begin{aligned}
\mathscr{C}\left(\bar{E} B, \bar{E} B^{\prime}\right) & =\mathscr{C}\left(\int^{A} \mathscr{B}(N A, B) \cdot E A, \int^{A^{\prime}} \mathscr{B}\left(N A^{\prime}, B^{\prime}\right) \cdot E A^{\prime}\right) \\
& \cong \int_{A}\left[\mathscr{B}(N A, B), \mathscr{C}\left(E A, \int^{A^{\prime}} \mathscr{B}\left(N A^{\prime}, B^{\prime}\right) \cdot E A^{\prime}\right)\right] \\
& \cong \int_{A}\left[\mathscr{B}(N A, B), \int^{A^{\prime}} \mathscr{B}\left(N A^{\prime}, B^{\prime}\right) \otimes \mathscr{F}\left(\mathscr{A}\left(A^{\prime},-\right), \mathscr{A}(A,-)\right)\right] \\
& \cong \int_{A}\left[\mathscr{B}(N A, B), \int^{A^{\prime}} \mathscr{B}\left(N A^{\prime}, B^{\prime}\right) \otimes \mathscr{A}\left(A, A^{\prime}\right)\right]
\end{aligned}
$$

by the representation theorem,

$$
\cong \int_{A}\left[\mathscr{B}(N A, B) \mathscr{B}\left(N A, B^{\prime}\right)\right]
$$


by the representation theorem,

$$
\cong \mathscr{B}\left(B, B^{\prime}\right) \text { if and only if } N \text { is dense. }
$$

\section{Appendix II}

The embedding of the promonoidal monad $\mathscr{T}$ into the monoidal biclosed monad $W \overline{\bar{F}}$ (see $\$ 3$ ) may be refined so that the embedding of $\mathscr{A}^{9}$ preserves certain colimits. This is achieved by considering the monoidal closure of the Isbell completion of $\mathscr{B}$ in $\left[\mathscr{B}^{\circ}, \mathscr{V}\right]$ (see Day (1974b) for notation).

Firstly, let $Z^{\circ}$ be the monoidal interior $\{z \in Z ; F \otimes z \in Z$ and $z \otimes F \in$ $Z$ for all $\left.F \in\left[\mathscr{B}^{\text {op }}, \mathscr{V}\right]\right\}$ of the class $Z$ of morphisms inverted by the Isbell conjugation functor:

$$
F \mapsto \int_{B}[F B, \mathscr{B}(B,-)]
$$

from $\left[\mathscr{B}^{\text {op }}, \mathscr{V}\right]$ to $[\mathscr{B}, \mathscr{V}]^{\text {op }}$. Denote $[\mathscr{B} \underset{\mathscr{T}}{\text { op }}, \mathscr{V}]$ by $\mathscr{C}$ and form $\mathscr{D}=\{H \in \mathscr{C}$; $\left.W(H) \perp Z^{\circ}\right\}$ as a full subcategory of $\mathscr{C}$.

6.1 Proposition. The category $\mathscr{D}$ is closed under limits and under exponentiation in $\mathscr{C}$.

Proof. Clearly $\mathscr{D}$ is closed under limits in $\mathscr{C}$. For all $G \in \mathscr{C}, H \in \mathscr{D}$, and $z \in Z^{\circ}$ we have that $\left[\mathscr{R}{ }^{\text {op }}, \mathscr{V}\right](W G \otimes z, W H)$ is an isomorphism, hence $C(\overline{\bar{F}}(W G \otimes z), H) \cong \mathscr{C}(\overline{\bar{F}} W G \otimes \overline{\bar{F}} z, H)$ are isomorphisms. This implies, by monadicity of $W \overline{\bar{F}}$, that $\mathscr{C}(G \otimes \overline{\bar{F}} z, H)$ is an isomorphism. Thus $\mathscr{C}(\overline{\bar{F}} z, G \backslash H) \cong\left[\mathscr{B}^{\text {op }}, \mathscr{V}\right](z, W(G \backslash H))$ are isomorphisms. Therefore we have $W(G \backslash H) \perp Z^{\circ}$ whence $G \backslash H \in \mathscr{D}$ for all $G \in \mathscr{C}$ and $H \in \mathscr{D}$. Similarly $H / G \in \mathscr{D}$ for all $G \in \mathscr{C}$ and $H \in \mathscr{D}$.

Under suitable hypotheses on $\mathscr{V}$ the category $\mathscr{D}$ is reflective in $\mathscr{C}$ and the reflection $R$ is necessarily monoidal (by 6.1 Proposition) when it exists. It is easy to verify that $\mathscr{D}$ is monadic over $\left[\mathscr{B}^{\text {op }}, \mathscr{V}\right]_{z^{\circ}}$ and contains $\mathscr{A}^{\mathscr{F}}$ as a full subcategory.

Now consider a colimit colim $K$ of a functor $K: \mathscr{K} \rightarrow \mathscr{A}^{\mathscr{g}}$. This gives a comparison transformation

in $\mathscr{C}$.

$$
\kappa: \operatorname{colim} \bar{E} K \rightarrow \bar{E}(\operatorname{colim} K)
$$

6.2 Proposition. If $E U: \mathscr{A}^{\mathscr{T}} \rightarrow\left[\mathscr{B}^{\text {op }}, \mathscr{V}\right]_{Z^{\circ}}$ preserves colim $K$ then $R \bar{E}: \mathscr{A}^{\mathcal{T}} \rightarrow \mathscr{D}$ preserves colim $K$.

Proof. The embedding $R \bar{E}$ preserves colim $K$ if and only if $\kappa$ is inverted by the reflection $R$. This is so if $k \perp H$ for all $H \in \mathscr{D}$. But $W_{\kappa} \in Z^{\circ}$ 
because $E U$ preserves colim $K$ by hypothesis. Thus, by definition of $\mathscr{D}$, $W \kappa \perp W H$ for all $H \in \mathscr{D}$. But $\left[\mathscr{B}^{\text {op }}, \mathscr{V}\right](W \kappa, W H)$ is an isomorphism for all $H \in \mathscr{D}$ iff $\mathscr{C}\left(\overline{\bar{F}} W_{\kappa}, H\right)$ is an isomorphism for all $H \in \mathscr{D}$. This implies that $\overline{\bar{F}} W \kappa$ is inverted by $R$, whence $\kappa$ is inverted by $R$ because $\overline{\bar{F}} \dashv W$ is monadic.

Therefore, under suitable hypotheses on $\mathscr{V}$, there exists an embedding $R \bar{E}$ of $\mathscr{A}^{s}$ which preserves all the colimits preserved by $E U: \mathscr{A}^{\mathscr{F}} \rightarrow\left[\mathscr{B}^{\text {of }}, \mathscr{V}\right]_{Z^{\circ}}$; this generalises the embedding theorem for closed categories discussed in [7] by the author. Note that, by the change-of- $\mathscr{V}$ universe procedure outlined in Day (1973), we may replace $\mathscr{V}$ by a larger category $\mathscr{W}$ with respect to which $\mathscr{A}$ and $\mathscr{A}^{\mathcal{F}}$ are both "small". This implies that the hypotheses necessary on $\mathscr{V}$ in order that $\mathscr{D}$ be reflective in $\mathscr{C}$ are always satisfied if $\mathscr{V}$ is replaced by $\mathscr{W}$ throughout.

\section{References}

J. Bénabou (1967), 'Introduction to bicategories', Reports of the Midwest Category Seminar I (Springer Lecture Notes, Vol. 47), 1-77.

B. J. Day and G. M. Kelly (1969), 'Enriched functor categories', Reports of the Midwest Category Seminar III (Springer Lecture Notes, Vol. 106), 178-191.

B. J. Day (1970a), 'On closed categories of functors', Reports of the Midwest Category Seminar IV (Springer Lecture Notes, Vol. 137), 1-38.

B. J. Day (1970b), 'Construction of biclosed categories', Ph.D. Thesis, University of New South Wales.

B. J. Day (1973), 'Note on monoidal localisation', Bull. Austral. Math. Soc. 8, 1-16.

B. J. Day (1974a), 'On closed categories of functors II', Category Seminar, Sydney 1972/73 (Springer Lecture Notes, Vol. 420), 20-53.

B. J. Day (1974b), 'An embedding theorem for closed categories', Category Seminar, Sydney 1972/73 (Springer Lecture Notes, Vol. 420), 55-64.

E. Dubuc (1970), Kan extensions in enriched category theory (Springer Lecture Notes, Vol. 145).

S. Eilenberg and G. M. Kelly (1966), 'Closed categories', Proc. Conference on Categorical Algebra, La Jolla, 1965 (Springer-Verlag, 1966), 421-562.

G. M. Kelly (1974), 'On clubs and doctrines', Category Seminar, Sidney 1972/73 (Springer Lecture Notes, Vol. 420), 181-256.

A. Kock (1970), 'Monads on symmetric monoidal closed categories', Archiv der Mathematik, Vol. XXI, $1-10$.

A. Kock (1971a), 'Closed categories generated by commutative monads', J. Austral. Math. Soc., $12,405-424$.

A. Kock (1971b), 'Bilinearity and cartesian closed monads', Math. Scand., 29, 161-174.

F. E. J. Linton (1969), 'Coequalisers in categories of algebras', Seminar on Triples and Categorical Homology Theory (Springer Lecture Notes, Vol. 80), 75-90.

R. H. Street (1972), 'The formal theory of monads', J. of Pure and Applied Algebra, 2, 149-168.

Department of Pure Mathematics,

University of Sydney,

N.S.W. 2006,

Australia. 